Editorial: Hospital Metropolitano

ISSN (impreso) 1390-2989 - ISSN (electrónico) 2737-6303

Edición: Vol. 28 No 3 (2020) Julio - Septiembre

DOI: https://doi.org/10.47464/MetroCiencia/vol28/3/2020/4-7

URL: https://revistametrociencia.com.ec/index.php/revista/article/view/79

Pág: 4-7

\title{
Quiste congénito del conducto nasolacrimal/dacrio- cistocele: causa de dacriocistitis no diagnosticada
}

\section{congenital cyst of nasolacrimal duct/dacriocistocele: cause of non-diagnosed dacriocistitis}

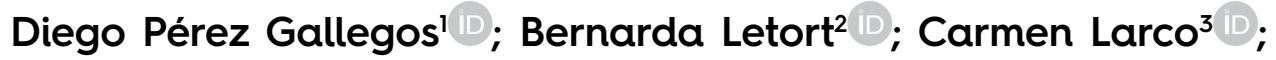 \\ Andrea Molinari ${ }^{4}$; Rodrigo Castrillón ${ }^{5}$; José Letort $^{6}$ \\ Departamento de Otorrinolaringología, Escuela de Medicina, Pontificia Universidad Católica del Ecuador, Quito, Ecuador ${ }^{1}$ \\ Universidad Internacional del Ecuador, Quito, Ecuador ${ }^{2}$ \\ Universidad Tecnológica Equinoccional, Quito, Ecuador \\ Oftalmología, Hospital Metropolitano, Quito, Ecuador \\ Centro de Otorrinolaringología, Hospital Metropolitano, Quito, Ecuador ${ }^{5,6}$
}

Recibido: 10/05/2020 Aceptado: 26/05/2020 Públicado: 01/09/2020

\section{RESUMEN}

Se presenta dos casos de pacientes menores de 5 meses los cuales durante las primeras semanas de vida presentan descarga ocular muco-purulenta de manera recurrente. Posteriormente en la endoscopia se encuentra en los dos casos masas quísticas intranasales dando un diagnóstico de dacriocistocele más quiste congénito del conducto nasolagrimal. El dacriocistocele es una causa muy rara de obstrucción del conducto nasal y más raro cuando viene acompañado de un quiste congénito del conducto nasolagrimal. Debido a su rareza y a su sintomatología muchas veces atípica resulta en un diagnóstico complicado para el otorrinolaringólogo y para el oftalmólogo. Palabras claves: Quiste congénito del conducto nasolacrimal, dacriocistocele, anomalías congénitas, aparato lacrimal.

\section{ABSTRACT}

Two cases of patients younger than 5 months are presented, who during the first weeks of life have recurrent muco-purulent ocular discharge. Subsequently, in endoscopy, in both cases, intranasal cystic masses were found, resulting in a diagnosis of dacryocystocele plus congenital cyst of the lacrimal duct. Dacryocystocele is an uncommon cause of nasal duct obstruction, but it becomes less common when accompanied by a congenital tear duct cyst. Due to its uncommonness and its often-atypical symptoms, it results in a complicated diagnosis for the ENT and the ophthalmologist.

Keywords: Congenital cyst of the nasolacrimal duct, dacryocystocele, congenital anomalies, lacrimal apparatus.

Diego Pérez Gallegos:

Bernarda Letort:

Carmen Larco:

Andrea Molinari:

Rodrigo Castrillón:

José Letort:

\section{IDs Orcid}

https://orcid.org/0000-0002-8075-507X https://orcid.org/0000-0001-9544-5173 https://orcid.org/0000-0002-2325-1115 https://orcid.org/0000-0003-0243-688X https://orcid.org/0000-0001-6206-1202 https://orcid.org/0000-0002-4039-6180

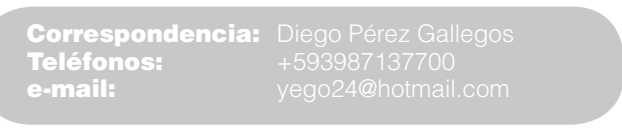




\section{INTRODUCCIÓN}

La obstrucción congénita del conducto nasolagrimal (OCNL) es una afección común que causa lagrimeo excesivo o descarga mucoide de los ojos, esto debido al bloqueo del sistema del conducto nasolagrimal' ${ }^{1}$. Sin embargo, la embriogénesis de la formación de un quiste congénito del conducto nasolagrimal se atribuye a la falla de la membrana de Hasner del sistema del conducto nasolagrimal para canular y debido la obstrucción proximal a nivel de la válvula de Rosenmuller².

La obstrucción en general del conducto nasolagrimal se presenta en $6 \%$ al $20 \%$ de los infantes menores de 1 año en todo el mundo quienes desarrollan alguna sintomatología de este trastorno ${ }^{3}$ y a menudo se resuelve sin necesidad de cirugía durante las primeras semanas de vida ${ }^{1,2,4}$, la presencia de dacriocistocele es extremadamente raro ${ }^{5}$, sin embargo, de estos pacientes aproximadamente $0.1 \%$ es secundaria al diagnóstico de dacriocistocele acompañado de quiste congénito del conducto nasolagrimal ${ }^{2}$.

Su diagnóstico es difícil para el otorrinolaringólogo (ENT), el oftalmólogo, pediatra y el radiólogo debido a su rareza y su sintomatología, que a veces es atípica o poco común en los recién nacidos ${ }^{3}$.

\section{CASO 1}

Paciente masculino de 4 meses de edad que desde sus primeras semanas de vida presenta epifora, posteriormente desarrolla descarga mucosa-purulenta del ojo derecho y edema infeccioso a nivel de saco lagrimal derecho a repetición. Sin embargo, la descarga mucosa mejoro con antibiótico tópico pero el edema azulado a nivel de canto medial del ojo derecho se mantuvo por lo que se inicio evaluaciones complementarias. El paciente no tenía síntomas respiratorios o dificultad respiratoria, examen ocular normal. La endoscopia transnasal demostró una masa quística unilateral bien delimitada a la altura del meato inferior derecho a nivel de la válvula de Hasner (Fig. 1A) por lo que el diagnóstico de Dacriocistocele más quiste intranasal en el meato inferior fue dado. Bajo anestesia general se realizó marsupialización endonasal y canalización lagrimal (Fig. 1B). Postoperatorio sin complicaciones, en controles de seguimiento no se ha observado recurrencia.


Figura 1. A. Vista endoscópica transquirúrgica de la fosa nasal derecha evidenciando un quiste intranasal ubicado bajo el meato inferior. B. Vista endoscópica transquirúrgica inmediatamente después de marsupialización.

Fuente: Los autores

\section{CASO 2}

Paciente femenina de 12 semanas de edad que presenta cuadro clínico de dacriocistitis supurativa crónica caracterizado por descarga ocular purulenta izquierda que se presenta de manera recurrente o resistente a medidas locales y tratamiento antibiótico, sin disnea. Se decide conjuntamente con Oftalmología programar canalización endoscópica bajo anestesia general, sin embargo, como hallazgo incidental durante canalización de sistema nasolacrimal se evidencia quiste intranasal en meato inferior izquierdo (Fig. 2), dando un diagnóstico de dacriocistocele más quiste congénito del conducto nasolagrimal. Se realizó marsupialización endonasal con evolución favorable. Postoperatorio sin complicaciones, en seguimiento presenta remisión de sintomatología. 

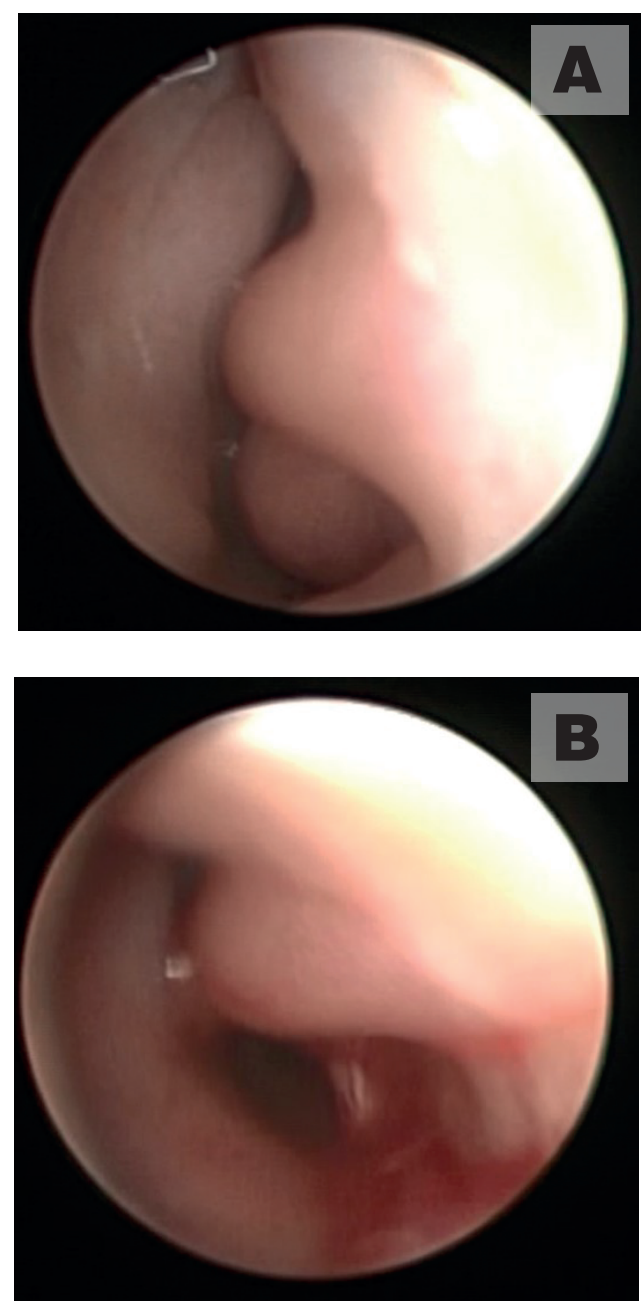

Figura 2. A. Vista endoscópica transquirúrgica demostrando la ocupación del meato inferior por un quiste intranasal en fosa nasal izquierda. B. Posterior a marsupialización.

\section{Fuente: Los autores}

\section{DISCUSIÓN}

La obstrucción mecánica de la porción distal de la válvula de Hasner causa un retorno hacia arriba del fluido por lo que los niños presentan epifora ${ }^{3}$. Además, esta entidad cuando no presenta infección está caracterizada por la aparición de una masa firme azulada sobre la región del conducto nasolacrimal específicamente bajo el tendón cantal medial5,6,7. El paciente presenta epifora, esto causa acumulación de líquido en el sistema de drenaje, inicialmente se llena de material mucoide que posteriormente puede infectarse y presentar descarga mucopurulenta o puede desarrollar celulitis ${ }^{7,8}$.

En casos raros puede existir presentación bilateral y el paciente puede presentar distrés respiratorio y requerir tratamiento urgente ${ }^{9}$, además se han reportado casos de asociación con atresia bilateral de coanas $^{10}$.
Para su diagnóstico es crucial la endoscopía nasal que demuestra la presencia de una masa quística intranasal que emerge del meato inferior en su región anterior ${ }^{11}$. El uso de ecografía puede confirmar la naturaleza del quiste. La tomografía computarizada muestra un masa regular y bien definida con una bolsa cantal en la esquina interna del ojo y una bolsa nasal en el saco lacrimal que a menudo empuja el cornete inferior contra el tabique ${ }^{12}$. La resonancia magnética es superponible, con la misma sensibilidad diagnóstica que la tomografía y permite una buena individualización del contenido de líquido y sus límites con la ventaja de no irradiar al paciente, además permite argumentar el diagnóstico diferencial: quiste dermoide, hemangioma capilar, encefalocele, meningoencefalocele en continuidad con espacios subaracnoideos y más raramente glioma, sarcoma o fibroma ${ }^{11,12}$.

Las opciones de tratamiento disponibles son la terapia conservadora, incluida la observación, el masaje del saco lagrimal, uso de antibióticos lo cual resuelve el $90 \%$ de los casos, el resto de pacientes requieren terapia invasiva ${ }^{13}$. Existe poco consenso con respecto al manejo óptimo o el momento oportuno, pero las recomendaciones actuales favorecen la intervención quirúrgica temprana debido al riesgo de complicaciones asociadas ${ }^{14}$.

Las modalidades de tratamiento habituales son la sonda nasolacrimal, la colocación de stents de silastic y la marsupialización quística endoscópica intranasal15,16.

En presencia de dacriocistocele y quiste intranasal es necesaria la realización de marsupialización seguido de sondeo del conducto nasolacrimal17,18,19. La técnica de marsupialización nasal describe el uso inicial de descongestión nasal, seguida de una incisión de la pared medial del quiste 8-10 mm a lo largo del eje vertical del conducto nasolagrimal. Se realiza una incisión horizontal antero-posterior 8-10 mm perpendicular a la primera, formando una incisión cruzada. Los contenidos del dacriocistocele se vacía a través de la incisión cruzada, además se puede utilizar microdebridador, una pinza ahuecada o de cocodrilo para atrapar el quiste ${ }^{14,20}$. Y se realiza una prueba con una sonda 45 para confirmar la apertura del quiste. La marsupialización del quiste intranasal es una modalidad efectiva de manejo para un dacriocistocele con buenos resultados a largo plazo ${ }^{20}$.

\section{CONCLUSIONES}

Ciertamente estos casos proveen una oportunidad para revisar esta rara anomalía lacrimal congénita y así sospechar el diagnóstico de quiste congénito del conducto nasolacrimal/dacriocistocele; a pesar de que puede ser bastante desafiante por las características clínicas, es importante detectarlos de ma- 
nera temprana porque esta condición puede causar dacriocistitis crónica que no resuelve con métodos convencionales y algunos casos tienden a presentar obstrucción nasal o dificultad respiratoria.

\section{CONTRIBUCIÓN DE LOS AUTORES}

Diego Pérez Gallegos: Concepción y diseño del trabajo; recolección y obtención de resultados; análisis e interpretación de datos; redacción del manuscrito.

Bernarda Letor: Concepción y diseño del trabajo; recolección y obtención de resultados; análisis e interpretación de datos; redacción del manuscrito.

Carmen Larco: Concepción y diseño del trabajo; recolección y obtención de resultados; análisis e interpretación de datos; redacción del manuscrito.

Andrea Molinari: Concepción y diseño del trabajo; recolección y obtención de resultados; análisis e interpretación de datos; redacción del manuscrito.

Rodrigo Castrilló: Concepción y diseño del trabajo; recolección y obtención de resultados; análisis e interpretación de datos; redacción del manuscrito.

José Letort: Concepción y diseño del trabajo; recolección y obtención de resultados; análisis e interpretación de datos; redacción del manuscrito.

\section{CONFLICTO DE INTERESES}

Los autores declararon no tener ningún conflicto de interés personal, financiero, intelectual, económico y de interés corporativo con el Hospital Metropolitano y los miembros de la revista MetroCiencia.

\section{REFERENCIAS BIBLIOGRÁFICAS}

1. Vagge A, Ferro Desideri L, Nucci P, Serafino M, Giannaccare G, Lembo A, et al. Congenital Nasolacrimal Duct Obstruction (CNLDO): A Review. Diseases [Internet]. 2018 Oct 22 [cited 2020 Mar 19];6(4):96. Available from: http://www.mdpi.com/2079-9721/6/4/96

2. Barham HP, Wudel JM, Enzenauer RW, Chan KH. Congenital nasolacrimal duct cyst/dacryocystocele: An argument for a genetic basis Allergy Rhinol [Internet]. 2012 May 3 [cited 2020 Abr 10];3(1):469. Available from: http://journals.sagepub.com/doi/full/10.2500/ ar.2012.3.0024

3. Lueder GT. The association of neonatal dacryocystoceles and infantile dacryocystitis with nasolacrimal duct cysts (an American ophthalmological society thesis). Trans Am Ophthalmol Soc [Internet] 2012 [cited 2020 Mar 15];110:74-93. Available from: /pmc/articles/ PMC3671363/?report=abstract
4. Davies R, Watkins WJ, Kotecha S, Watts $\mathbf{P}$. The presentation, clinical features, complications, and treatment of congenital dacryocystocele. Eye [Internet]. 2018 Mar 1 [cited 2020 Abr 19];32(3):522-6. Available from: www.nature.com/eye

5. Shekunov J, Griepentrog GJ, DiehI NN, Mohney BG. Prevalence and clinical characteristics of congenital dacryocystocele. J AAPOS [Internet]. 2010 Oct 1 [cited 2020 Abr 19];14(5):417-20. Available from: http://www.jaapos.org/article/S1091853110003745/fulltext

6. Wong RK, Vander Veen DK. Presentation and Management of congenital dacryocystocele. Pediatrics [Internet]. 2008 Nov [cited 2020 Abr 19];122(5). Available from: https://pubmed.ncbi.nlm.nih. gov/18955412/

7. Shashy RG, Durairaj V, Holmes JM, Hohberger GG, Thompson DM, Kasperbauer JL. Congenital dacryocystocele associated with intranasal cysts: Diagnosis and management. Laryngoscope [Internet]. 2003 Jan 1 [cited 2020 Abr 19];113(1):37-40. Available from: https:// pubmed.ncbi.nlm.nih.gov/12514379/

8. Cavazza S, Laffi GL, Lodi L, Tassinari G, Dall'Olio D. Congenital dacryocystocele: diagnosis and treatment. Acta Otorhinolaryngol Ital [Internet]. 2008 Dec [cited 2020 Abr 19];28(6):298-301. Available from: /pmc/articles/PMC2689544/?report=abstract

9. Ismi O, Bozkurt FM, Icme G, Eti C, Sari A. A rare cause of intermittent respiratory distress and epiphora in the newborn: Congenital dacryocystocele. Gland Surg. 2017;6(1):114-8.

10. Neto JFL, Kuhl G, Smith MM, de Souza PA, Vieira LR. Association between congenital nasolacrimal duct cyst and bilateral choanal atresia. Braz J Otorhinolaryngol [Internet]. 2011 [cited 2020 Abr 19];77(2):267. Available from: http://www.bjorl.org/

11. Al-Faky YH. Nasal endoscopy in the management of congenital nasolacrimal duct obstruction. Saudi J Ophthalmol. 2014 Jan 1;28(1):6-11.

12. Braun JJ, Debry C, Donato L, Riehm S. Kyste lacrymonasal congénital : Une entité radioclinique à ne pas méconnaître. Arch Pediatr. 2016 Feb 1;23(2):192-6.

13. Schnall BM. Pediatric nasolacrimal duct obstruction. Curr Opin Ophthalmol [Internet]. 2013 Sep [cited $2020 \mathrm{Abr} 19] ; 24(5): 421-4$. Available from: http://journals.Iww.com/00055735-201309000-00008

14. Zhao NW, Chan DK. Awake bedside nasal endoscopy for primary management of neonatal dacryocystoceles with intranasal cysts. Int J Pediatr Otorhinolaryngol. 2019 Aug 1;123:93-6.

15. Natesh BG, Patil S, Nilssen E, Maclean H. Endonasal microdebrider assisted excision of congenital intranasal nasolacrimal duct cyst or inferior mucocoele. Indian J Otolaryngol Head Neck Surg [Internet]. 2016 Dec 19 [cited 2020 Nov 19];68(1):115-7. Available from: https:// link.springer.com/article/10.1007/s12070-015-0948-y

16. Avram E. Insights in the treatment of congenital nasolacrimal duct obstruction. Rom J Ophthalmol. 2017;61(2):101-6.

17. Dogan E, Yüksel NG, Ecevit MC, Yaman A, Berk AT, Sütay S. Microdebrider assisted endoscopic marsupialization of congenital intranasal nasolacrimal duct cysts. Int J Pediatr Otorhinolaryngol [Internet]. 2012 Apr [cited 2020 Nov 20];76(4):488-91. Available from: https:// pubmed.ncbi.nlm.nih.gov/22277269/

18. Sarbajna T, Takahashi Y, Paula Valencia MR, Ana-Magadia MG, Ishikawa E, Kakizaki H. Dacryoendoscopy-assisted nasal endoscopic marsupialization for congenital dacryocystocele. Int J Pediatr Otorhinolaryngol. 2018 Dec 1;115:54-7.

19. Suzuki Y, Omura K, Otori N, Tanaka Y. Endonasal marsupialisation of a congenital intranasal nasolacrimal duct cyst [Internet]. Vol. 12, BMJ Case Reports. BMJ Publishing Group; 2019 [cited 2020 Nov 20]. p. 228284. Available from: http://casereports.bmj.com/

20. Ali MJ, Singh S, Naik MN. Long-term outcomes of cruciate marsupialization of intra-nasal cysts in patients with congenital dacryocele. Int J Pediatr Otorhinolaryngol. 2016 Jul 1;86:34-6. 